\title{
CLASSIFICATION THEORY FOR HARDY CLASSES OF ANALYTIC FUNCTIONS
}

\author{
BY DENNIS A. HEJHAL ${ }^{1}$
}

Communicated by Paul J. Cohen, March 19, 1971

I. Introduction. Suppose that $W$ is an open Riemann surface. Denote by $A(W)$ and $M(W)$ the families of single-valued analytic and meromorphic functions on $W$, respectively. The Hardy class $H_{p}(W)$, for $0<p<\infty$, is the family of all $f \in A(W)$ for which $|f|^{p}$ admits a harmonic majorant on $W$. Let $A B(W)$ be the family of all bounded $f \in A(W)$. Denote by $M B^{*}(W)$ the family of all $f \in M(W)$ such that $\ln ^{+}|f|$ admits a superharmonic majorant on $W$. Write $A B^{*}(W)=A(W) \cap M B^{*}(W)$. We shall write $W \in O_{p}, O_{A B}, O_{A B^{*}}, O_{M B^{*}}$ whenever $H_{p}(W), A B(W), A B^{*}(W), M B^{*}(W)$, respectively, reduces to the constant functions. Finally, as usual, $W \in O_{G}$ iff $W$ is parabolic.

Now, as is readily verified, $A B(W) \subseteq H_{p}(W) \leqq H_{q}(W) \subseteq A B^{*}(W)$ $\subseteq M B^{*}(W)$ for $0<q<p<\infty$. It follows that

$$
O_{G} \leqq O_{M B^{*}} \leqq O_{A B^{*}} \leqq \bigcap_{q>0} O_{q} \leqq O_{p}^{-} \leqq O_{p} \leqq O_{p}^{+} \leqq \underset{q<\infty}{\cup} O_{q} \leqq O_{A B},
$$

where $O_{p}^{-}=\mathrm{U}\left\{O_{q} \mid 0<q<p\right\}, O_{p}^{+}=\bigcap\left\{O_{q} \mid p<q<\infty\right\}, 0<p<\infty$. It is known that all of these inclusions are strict in the case of arbitrary Riemann surfaces (see Heins [3, pp. 34-50] and Sario-Nakai [7, pp. 276-280]). The appropriate constructions are Myrberg type surfaces and hence of infinite genus.

If one now restricts $W$ to be of finite genus, the situation changes. First of all, it is now known that $O_{G}=O_{M B^{*}}=O_{A B^{*}}$ (see Sario-Nakai $\left[7\right.$, p. 280]). Further, Heins [3, pp. 50-51] showed next that $O_{G}<O_{1}$ $\leqq O_{A B}$. Aside from these facts, the classification scheme for Hardy classes for Riemann surfaces of finite genus, and thus for plane domains, has remained an open question (see Heins $[3$, p. 50] and Rudin [6, p. 49]).

In one of our recent projects, we found a number of results on function-theoretic null-sets and classification theory for $H_{p}$ classes. In this note we wish to present some of these results. Included will be a partial, though highly suggestive, answer to the open question mentioned

AMS 1970 subject classifications. Primary 30A44, 30A48, 30A78, 31C15.

Key words and phrases. Open Riemann surfaces, Hardy $H_{p}$ classes, classification theory, function-theoretic null-sets, Riesz potential, Nevanlinna-Frostman theorem.

${ }^{1}$ Supported by an NSF Traineeship at Stanford University. 
above. A more detailed development will appear at a later date.

I wish to thank Professor Halsey Royden for his encouragement and helpful discussions.

II. On removable singularities. Let $S$ denote the sphere and $X$ the finite plane. Suppose that $E$ is a bounded closed totally disconnected subset of $S$. We shall write $E \in N_{p}$ iff $H_{p}(V)=H_{p}(V-E)$ for every subdomain $V$ of $S$ for which $E \subseteq V$. $E \in N_{B}$ and $E \in N_{M B^{*}}$ are defined similarly using $A B$ and $M B^{*}$, respectively. Let $E \in N_{G}$ iff $\operatorname{Cap}(E)=0$. Finally, write $E=\Re\left[\lambda_{1}, \cdots, \lambda_{n}\right]$ iff $E$ is concentrated entirely on an $n$-star formed by $n$ rays emanating from an origin out to $\infty$ such that the successive angles are $\pi \lambda_{1}, \cdots, \pi \lambda_{n}, n \geqq 2, \lambda_{1}+\cdots+\lambda_{n}=2$.

The proofs of the following three theorems involve a combination of techniques from classical potential theory and classification theory.

Theorem 1. If $E \in N_{G}$, then $E \in N_{M B^{*}}$, and conversely.

THEOREM 2. The following are equivalent. (i) $E \in N_{p}$; (ii) $S-E \in O_{p}$; and (iii) $H_{p}(V-E)=H_{p}(V)$ for some subdomain $V$ of $S$ for which $E \subseteq V$.

TheOREM 3. Suppose that $E_{1}, \cdots, E_{n}$ are mutually disjoint and in $N_{p}$. Then, $E=\bigcup\left\{E_{k} \mid 1 \leqq k \leqq n\right\} \in N_{p}$.

The following theorem is a generalization of a result in Heins [3, p. $50]$.

TheOREM 4. Let $\Gamma_{1}, \cdots, \Gamma_{n}$ be a finite number of disjoint analytic Jordan arcs. Let $E_{1}, \cdots, E_{n}$ be bounded closed totally disconnected subsets of $S, E_{j} \leqq \Gamma_{j}, 1 \leqq j \leqq n$. Suppose that all $E_{j} \in N_{B}$. Then, $E=\bigcup\left\{E_{j} \mid 1 \leqq j \leqq n\right\} \in N_{1}$.

This theorem is proved by introduction of appropriate local analytic coordinates near the $\Gamma_{k}$ and by use of classical boundary properties of $H_{p}$ functions as presented, for example, in Golusin [2, pp. 345-366] and Privalov [5, pp. 53-83].

The next theorem can be effectively used as a fundamental lemma in the study of the $H_{p}$ classification. Its proof is in part similar to that of Theorem 4 and involves a study of the $H_{p}$ spaces corresponding to the sectors.

Theorem 5. Suppose that $E \in K\left[\lambda_{1}, \cdots, \lambda_{n}\right]$ and $E \in N_{B}$. Let $\lambda=\min \left\{\lambda_{j} \mid 1 \leqq j \leqq n\right\}$ and $1 \leqq p<\infty$. Suppose that $f \in H_{p}(S-E)$. Then,

$$
f(z)=\sum_{k=0}^{\infty} c_{k} z^{-k}, \quad 0<|z| \leqq \infty,
$$


with $c_{k}=0$ for all $k \geqq M_{p}$, where $M_{1}$ is the least integer $\geqq 1 / \lambda$ and $M_{p}$, $p>1$, is the least integer $>1 / p \lambda$. Thus, the complex linear space $H_{p}(S-E)$ is finite-dimensional.

Of course, Theorem 5 with $n=2, \lambda_{1}=\lambda_{2}=1$, is essentially Theorem 4 .

The last two theorems of this section will serve to show that Theorem 3 is in some strong sense best possible. The proofs depend upon constructions that arise in the following section.

Theorem 6. Let $1 \leqq p<\infty$. Suppose that $E_{n} \in N_{p}$ for all $n \geqq 1$ and that the $E_{n}$ are mutually disjoint. Suppose too that $E=U\left\{E_{n} \mid 1 \leqq n<\infty\right\}$ is a bounded closed subset of $S$. Then, it can happen that $E \notin N_{q}$, all $0<q<\infty$.

Theorem 7. Suppose that $E_{1} \in N_{p}$ and $E_{2} \in N_{p}$ for some $0<p<\infty$. It can then happen that $E_{1} \cup E_{2} \oplus N_{p}$ although $E_{1} \cap E_{2}$ consists of a single point.

We can conclude from these theorems that function-theoretic nullsets of type $N_{p}$ do not have the properties expected by analogy with $N_{G}$ and $N_{B}$.

III. On the $H_{p}$ classification. Our main result on the $H_{p}$ classification is the following.

THEOREM 8. For plane domains the following classification scheme holds:

$$
\begin{aligned}
O_{G} & \leqq O_{1}^{-}<O_{1} \leqq O_{3 / 2}^{-}<O_{3 / 2} \leqq O_{2}^{-}<O_{2} \leqq O_{5 / 2}^{-}<O_{5 / 2} \\
& \leqq O_{3}^{-}<O_{3} \cdots<\bigcup_{0<p<\infty}^{\bigcup} O_{p}<O_{A B}
\end{aligned}
$$

The most difficult portion of the proof consists of showing that $O_{1}^{-}<O_{1}$. This is handled by use of Riesz $\alpha$-potentials and the associated potential theory as in Carleson [1, pp. 14-39]. The appropriate construction is a symmetric bounded closed totally disconnected subset $E$ of the real axis such that $E \in N_{B}$ and $1 / z \in H_{p}(S-E)$ for all $0<p<1$; see Theorem 4. Inequality $O_{k / 2}^{-}<O_{k / 2}$ for $k \geqq 3$ is now proved by use of transformation $w=z^{2 / k}$.

Because of its rather surprising simplicity, we will now give the proof of inequality $\mathrm{U}_{0<p<\infty} O_{p}<O_{A B}$. We need the following generalization of a classical theorem of Nevanlinna-Frostman.

Lemma. Let $D$ be a subdomain of $S, D \notin O_{G}$. Let $K$ be a bounded closed subset of $S$ with $\operatorname{Cap}(K) \neq 0$. Suppose that $f \in M(D)$ and $f[D] \cap K$ is void. Then, $f \in M B^{*}(D)$. 
By now, this result is reasonably well known. See, for example, Heins [4, pp. 426-428], Rudin [6, pp. 48-49], or Sario-Noshiro [8, p. 92].

Take any closed totally disconnected set $A$ on the unit circle such that:

(i) the linear measure of $A$ is 0 ;

(ii) $\operatorname{Cap}(A) \neq 0$;

(iii) $\operatorname{diam}(A)$ is very small;

(iv) $A$ is symmetric relative to 1 ;

(v) $1 \notin A$.

For instance, $A$ can be a Cantor set. Consider the set $F_{1} \equiv \log (\log A)$ $\leqq X$ in the $t$-plane. The set $F_{1}$ has a number of properties: (i) $F_{1}$ is bounded away from 0 ; (ii) $F_{1} \subseteq\{t \mid \operatorname{Re}(t)>c\}$, some $-\infty<c<0$; (iii) $F_{1}$ is concentrated on the lines $\operatorname{Im}(t)=(2 n+1) \pi / 2, n$ integral; (iv) $\infty$ is a cluster point of $F_{1}$. Form the image of $F_{1}$ under $z=1 / t$ and adjoin $\{0\}$ to get a bounded closed totally disconnected subset $E_{1}$ of $S$. At once, $E_{1} \in N_{B}-N_{G}$ (see Sario-Oikawa [9, pp. 289, 291]). Let $W_{1}=S-E_{1}$.

For $z \in W_{1}$, define $f_{1}(z)=\exp \left(e^{1 / z}\right)$ and $f_{2}(z)=1 / f_{1}(z)$ omits set $A$ for $z \in W_{1}$. Hence, by the Lemma, $f_{1} \in A B^{*}\left(W_{1}\right)$. Because $M B^{*}\left(W_{1}\right)$ is a field, $f_{2} \in A B^{*}\left(W_{1}\right)$. Let $\chi_{j}$ be the least harmonic majorant of $\ln ^{+}\left|f_{j}\right|$ on $W_{1}$ for $j=1,2$.

Proceed similarly with regard to the set $F_{2} \equiv \log (i \log A) \leqq X$. Here we let $W_{2}=S-E_{2}$ and define $f_{3}(z)=\exp \left(i e^{1 / z}\right), f_{4}(z)=1 / f_{3}(z)$ for $z \in W_{2} . f_{3}(z)$ then omits the set $A$ for $z \in W_{2}$. It follows that $f_{3}$ and $f_{4}$ are in $A B^{*}\left(W_{2}\right)$. Let $\chi_{j}$ be the least harmonic majorant of $\ln ^{+}\left|f_{j}\right|$ on $W_{2}$ for $j=3,4$.

Define $W=S-E_{1} \cup E_{2}$. At once, $E_{1} \cup E_{2} \in N_{B}$. Since $\sum_{j=1}^{4} \ln ^{+}\left|f_{j}\right|$ $\leqq \sum_{j=1}^{4} \chi_{j}(z), z \in W$, we find that $e^{1 / z} \in H_{1}(W)$. But, then, $e^{1 / p z}$ $\in H_{p}(W)$ for $0<p<\infty$. Hence, $W \in O_{A B}-\cup_{0<p<\infty} O_{p}$.

The proof of Theorem 6 also follows from this construction. In addition, the following weak form of Theorem 8 can be proved by use of just the same simple method and some simple changes of coordinate:

$$
O_{G} \leqq O_{1 / 2}<O_{1}<O_{2}<O_{4}<O_{8}<O_{16}<\cdots<\underset{0<p<\infty}{\bigcup} O_{p}<O_{A B}
$$

IV. Concluding remarks. Theorem 8 suggests quite strongly that for plane domains $O_{p}^{-}<O_{p}<O_{p}^{+}$when $1 \leqq p<\infty$. The case $0<p<1$ remains somewhat mysterious, however. The main reason for this seems to be the distinct lack of nontrivial techniques for the study of $H_{p}$ classes when $0<p<1$ (see Heins [3]). 


\section{REFERENCES}

1. L. Carleson, Selected problems on exceptional sets, Van Nostrand Math. Studies, no. 13, Van Nostrand, Princeton, N. J., 1967. MR 37 \#1576.

2. G. M. Golusin, Geometrische Funktionentheorie, Hochschulbücher für Mathematik, Band 31, VEB Deutscher Verlag der Wissenschaften, Berlin, 1957. MR 19, 735 .

3. M. Heins, Hardy classes on Riemann surfaces, Lecture Notes in Math., no. 98, Springer-Verlag, Berlin and New York, 1969. MR 40 \#338.

4. - Lindelöfian maps, Ann. of Math. (2) 62 (1955), 418-446. MR 17, 726.

5. I. I. Privalov, Randeigenschaften analytisher Funktionen, Hochschulbücher für Math., Band 25, VEB Deutscher Verlag, Berlin, 1956. MR 18, 727.

6. W. Rudin, Analytic functions of class $H_{p}$, Trans. Amer. Math. Soc. 78 (1955), 46-66. MR 16, 810.

7. L. Sario and M. Nakai, Classification theory of Riemann surfaces, SpringerVerlag, Berlin, 1970.

8. L. Sario and K. Noshiro, Value distribution theory, Van Nostrand, Princeton, N. J., 1966. MR 35 \#6833.

9. L. Sario and K. Oikawa, Capacity functions, Die Grundlehren der math. Wissenschaften, Band 149, Springer-Verlag, Berlin and New York, 1969. MR 40 \#7441.

Stanford University, Stanford, California 94305 